BRX TH-680

CALT-TH 2014-147

\title{
Unconstrained canonical action for, and positive energy of, massive spin 2
}

\author{
S. Deser \\ Walter Burke Institute for Theoretical Physics, \\ California Institute of Technology, Pasadena, CA 91125; \\ Physics Department, Brandeis University, Waltham, MA 02454 \\ deser@brandeis.edu
}

\begin{abstract}
Filling a much-needed gap, we exhibit the $D=4$ Fierz-Pauli (FP) massive $s=2$ action, and its - manifestly positive - energy, in terms of its $2 s+1=5$ unconstrained helicity $(2,1,0)$ excitations, after reducing and diagonalizing the troublesome helicity- 0 sector.
\end{abstract}

\section{Introduction}

We begin with an apologia, since there is nothing still not known about the FP model [1]. However, there seems to be no published derivation 1 from its covariant and highly constrained form to the final unconstrained canonical action in terms of its $2 s+1=5$ helicity $( \pm 2, \pm 1,0)$ components. That form will displays that each mode propagates correctly and has manifestly positive energy, in the usual $L=p \dot{q}-H, H=\frac{1}{2}\left[p^{2}+q\left(-\nabla^{2}+m^{2}\right) q\right]$ form. While [1] realized that positive energy was essential, it was displayed rather opaquely; a subsequent formulation [3] was likewise less than transparently presented (and contains distracting typos). Indeed, proper use of the constraints is not altogether trivial, making the correct process instructive as a (minor) exercise in (free) field theory.

Historically, it was not until FP's 1939 work that the problem of representing massive spins $>1$ by tensor fields - involving many more than $2 s+1$ components - was raised, let alone solved. Given the current interest in massive gravity (mGR) with Einstein kinetic terms plus non-derivative mass terms involving a fixed, say flat, background, our summary may be useful. lndeed, this is a good place to note that-contrary to statements in the mGR literature - the mass terms destroy the whole (ADM) asymptotic energy formulation of GR as a $2 D$ surface integral at spatial infinity, just as the Coulomb asymptotic integral that counts total charge is lost in massive (Proca) vector

\footnotetext{
${ }^{1}$ This was actually done [2] for the more general case of the system embedded in dS, rather than flat, space; however that derivation involved (an even number of) steps using inverse powers of $\Lambda$, hence singular and not applicable here; its final result of course does limit to ours. It was also shown in detail here that the helicity 0 mode can be removed by suitably tuning $m^{2} / \Lambda$ in $\mathrm{dS}$.
} 
theory: the Newtonian/Coulomb fields decay much too fast there for these integrals to contribute at all.

\section{The Derivation}

The action and field equations of the theory are the sum of linearized GR and the -unique-mass term that eliminates the 6th, ghost helicity 0, degree of freedom (DoF). We work throughout in 1st order, $3+1$, canonical form, which simplifies the procedure and indeed starts directly in terms of the 6 conjugate pairs $\left(\pi^{i j}, h_{i j}\right)$ rather than the 10 covariant $h_{\mu \nu}$. The action is (see e.g., [2])

$$
\begin{aligned}
I & =\int d^{4} x\left\{\pi^{i j} \dot{h}_{i j}-H(\pi, h)\right\} \\
H & ={ }^{3} R_{Q}+\left(\pi_{i j}^{2}-\frac{1}{2} \pi^{2}\right)+4 n R_{0}+2 N_{i} \partial_{j} \pi^{i j}+\frac{1}{4} m^{2}\left(h_{i j}^{2}-h_{i i}^{2}-4 n h_{i i}-2 N_{i}^{2}\right),
\end{aligned}
$$

where (under the integral)

$$
{ }^{3} R_{Q}=\frac{1}{2} h_{i j} G_{i j}^{L}=-\frac{1}{4}\left[h_{\mathrm{TT}} \nabla^{2} h_{\mathrm{TT}}-h_{\mathrm{T}} \nabla^{2} h_{\mathrm{T}}\right], \quad R_{0}=\left(m^{2}-\nabla^{2}\right) h_{\mathrm{T}}+m^{2} h_{\mathrm{L}}
$$

$n \sim \frac{1}{2} h_{00}$ is a Lagrange multiplier enforcing the linear constraint $R_{0}=0$, while $N_{i}=h_{0 i}$ becomes an auxiliary field to be eliminated by completing squares, leaving only the six $(\pi, h)$ pairs - indeed our whole process consists of juggling quadratic forms. Finally, we recall that the linearized 3D Einstein tensor $G_{i j}^{L}\left(h_{l m}\right)$ is both identically conserved and independent of the longitudinal, gauge, parts of $h_{l m}$; The second ingredient, essential to the separation of the various helicity DoF in (1), is the usual orthogonal decomposition of any symmetric 3-tensor,

$$
S_{i j}=S_{i j}^{\mathrm{TT}}+\frac{1}{2}\left(\delta_{i j}-\hat{\partial}_{i} \hat{\partial}_{j}\right) S^{\mathrm{T}}+\left[\hat{\partial}_{j} S_{i}^{\mathrm{T}}+\hat{\partial}_{j} S_{i}^{\mathrm{T}}\right]+\hat{\partial}_{i} \hat{\partial}_{j} S^{\mathrm{L}}, \quad \partial_{i} S_{i}^{\mathrm{T}} \equiv 0, \quad \hat{\partial}_{i} \equiv \partial_{i} / \sqrt{\nabla^{2}}
$$

Completing squares in (1) removes the $N_{i}$ dependence of $H$ in favor of adding the term $2 m^{-2}\left(\partial_{j} \pi^{i j}\right)^{2}$ to $H$. There remains the elimination of the $R_{0}$ constraint, hence of one linear combination of the two helicity $0(T, L)$ modes. It will be equally essential to use $\dot{R}_{0}=0$ to further eliminate one combination of their conjugate momenta $\left(\pi^{\mathrm{T}}, \pi^{\mathrm{L}}\right)$ using $\dot{h} \sim \pi$; constraints "strike twice" in our 1st order form, since they are valid for all times. 2 .

The task before us then is to decompose (1) into a sum of three - non- interacting - orthogonal, two DoF sectors: Helicity $\pm 2(\mathrm{TT})$, helicity \pm 1 " $T_{i}$ ", and the $(T, L)$ helicity- 0 . The latter's Hamiltonian is the source of difficulty, being a priori non-positive before using the $R_{0}(T, L)=0$ constraint. To keep the discussion compact, we first dispose of the helicity $>0$ sectors: that of TT is trivial to obtain, being unconstrained; we simply add up the TT terms in (1); dropping "TT",

\footnotetext{
${ }^{2}$ That the original number, 6 , of $\pi^{i j} \dot{h}_{i j}$ kinetic terms decreases by one for every constraint is just Darboux's theorem on quadratic forms; in massless theory there are 4 constraints, leaving just the two $\left(\pi^{\mathrm{TT}}, q^{\mathrm{TT}}\right)$ pairs. We will see this more explicitly below.
} 
we have

$$
L=\pi^{i j} \dot{h}_{i j}-H \quad H=\pi_{i j}^{2}+\frac{1}{4}\left(h_{i j, k}^{2}+m^{2} h_{i j}^{2}\right) \geq 0
$$

Note that $H$ vanishes only for TT vacuum, $\pi=0=h$. The same is true of the transverse vector $\left(T_{i}\right)$ part, though it still requires some field redefinitions to achieve the same final form; here (1) also easily yields (omitting " $T_{i}$ ")

$$
H=\pi^{2}+2 m^{-2}\left(\pi_{, j}^{i j}\right)^{2}+\frac{1}{2} m^{2} h^{2} \geq 0 ;
$$

while not (yet) very pretty, this $\mathrm{H}$ is also positive and vanishes at $\left(T_{i}\right)$ vacuum, a result unaffected by the further field redefinitions required to reach the final $p \dot{q}-H$ form; we outline the process in the Appendix. [Recall, however, that correct energy functional form is only reached when the " $(p, q)$ " variables are redefined to ensure that the associated kinetic, " $p \dot{q} "$, term is itself free of unwanted numerical coefficients.]

We now face the final, $H(T, L)$, sector, where $R_{0}$ must be used - twice. There,

$$
\begin{aligned}
& I[T, L]=\int d^{4} x\left[\frac{1}{2} \pi^{\mathrm{T}} \dot{h}_{\mathrm{T}}+\pi^{\mathrm{L}} \dot{h}_{\mathrm{L}}-V\left(h_{\mathrm{T}}, h_{\mathrm{L}}\right)-K\left(\pi^{\mathrm{T}}, \pi^{\mathrm{L}}\right)\right], \\
& 4 V(h) \equiv h_{\mathrm{T}}\left(\nabla^{2}-m^{2}\right) h_{\mathrm{T}}-2 h_{\mathrm{T}} h_{\mathrm{L}}, \quad K(\pi) \equiv \frac{1}{2}\left(\pi^{\mathrm{L}}\right)^{2}-2 \pi^{\mathrm{T}} \pi^{\mathrm{L}}+2 \pi^{\mathrm{L}}\left(-m^{-2} \nabla^{2}\right) \pi^{\mathrm{L}} .
\end{aligned}
$$

We now show that both potential and kinetic parts of $H$ are positive, using the $R_{0}$ constraint and its time derivative respectively. Eliminating $h_{\mathrm{L}}$ yields

$$
V\left(h_{\mathrm{L}}, h_{\mathrm{T}}\right)=\frac{3}{8}\left[\left(h_{\mathrm{T}, i}\right)^{2}+m^{2} h_{\mathrm{T}}^{2}\right] \geq 0 ;
$$

again, $V$ only vanishes at vacuum, $h_{\mathrm{T}}=0$. Next we find the $\dot{R}_{0}=0$ constraint between $\pi^{\mathrm{T}}$ and $\pi^{\mathrm{L}}$ : The two field equations for $\pi \sim \dot{h}$ obtained by varying (6) w.r.t. the $\pi$ are

$$
\dot{h}_{\mathrm{L}}-\pi^{\mathrm{L}}+\pi^{\mathrm{T}}+4 m^{-2} \nabla^{2} \pi^{\mathrm{L}}=0, \quad \dot{h}_{\mathrm{T}}+2 \pi^{\mathrm{L}}=0 .
$$

Taking their appropriate vanishing linear combination, we learn that

$$
m^{2} \pi^{T}=\left(-2 \nabla^{2}-m^{2}\right) \pi^{\mathrm{L}}
$$

hence finally

$$
K\left(\pi^{\mathrm{L}}, \pi^{\mathrm{T}}\right) \rightarrow K\left(\pi^{\mathrm{L}}\right)=\frac{3}{2}\left(\pi^{L}\right)^{2} \geq 0 .
$$

We have now established $E \geq 0$ for the full theory, but one task is still to be completed: putting the helicity action into exact $p \dot{q}-H(p, q)$ form. Even before this is done, one can already see that the (second order) field equations are uniformly $\left(\square-m^{2}\right) h=0$, but it is an amusing exercise as well as a check on the result - to do so. Using (9), it is easy to translate the $(T, L)$ sector's 
$\pi^{\mathrm{L}} \dot{h}_{\mathrm{L}}+\frac{1}{2} \pi^{\mathrm{T}} \dot{h}_{\mathrm{T}}$ into $\pi^{\mathrm{L}} \dot{h}_{\mathrm{T}}$ form. At this penultimate point,

$$
L(T, L)=-\frac{3}{2} \pi^{\mathrm{L}} \dot{h}_{\mathrm{T}}-\frac{3}{2}\left[\left(\pi^{\mathrm{L}}\right)^{2}+\frac{1}{4}\left(h_{T, i, i}\right)^{2}+\frac{1}{4} m^{2} h_{\mathrm{T}}^{2}\right] ;
$$

the obvious rescaling $(\pi, h) \rightarrow \sqrt{2 / 3}(-\pi, h)$ achieves the desired final canonical form of the helicity 0 sector,

$$
L(0)=\pi \dot{h}-H(\pi, h), \quad H \equiv \pi^{2}+\frac{1}{4} h\left(-\nabla^{2}+m^{2}\right) h .
$$

Together with the vector mode in the Appendix, then, the total action is

$$
L=\sum_{A=1}^{5} p^{A} \dot{q}_{A}-\frac{1}{2}\left[\left(p^{A}\right)^{2}+q_{A}\left(-\nabla^{2}+m^{2}\right) q_{A}\right],
$$

after the (cosmetic) rescaling $\pi \rightarrow p^{A} / \sqrt{2}, h \rightarrow q_{A} \sqrt{2}$.

\section{Summary}

The physical correctness of the massive $s=2 \mathrm{FP}$ model has been displayed: each of its $2 s+1=5$ helicity excitations obey $\left(\square-m^{2}\right) h=0, E \geq 0$.

\section{Appendix: The helicity \pm 1 sector}

We consider here the remaining, helicity 1 , subspace involving only the " $T_{i}$ " parts of $(\pi, h)$ in $(1)$. Clearly, neither $h G_{\mathrm{L}}(h)$ nor the $R_{0}$ constraint involve $h_{\mathrm{T} i}$; only the mass term does: it contains $\frac{1}{2} m^{2}\left(h_{\mathrm{T} i}\right)^{2}$. Its $\pi$ sector involves $\left(\pi_{i, j}^{\mathrm{T}}\right)^{2}$ as well as the quadratic term $2 N_{i}^{\mathrm{T}} \partial_{j} \pi^{i j}$. Using the $\frac{1}{2} m^{2}\left(N_{i}^{\mathrm{T}}\right)^{2}$ from the mass term, we complete the square to leave a net contribution $m^{-2}\left(\pi^{i j}{ }_{j}\right)^{2} \sim$ $2 m^{-2}\left(\pi_{i}^{\mathrm{T}} \nabla^{2} \pi_{i}^{\mathrm{T}}\right)$ there. At this point, then, dropping the $T_{i}$ indices, we find

$$
L\left(T_{i}\right)=-2 \pi \dot{h}-\frac{1}{2}\left[m^{2} h^{2}+4 m^{-2} \pi\left(m^{2}-\nabla^{2}\right) \pi\right]
$$

the necessary redefinition is obvious:

$$
\pi \rightarrow-\frac{1}{2} m\left(m^{2}-\nabla^{2}\right)^{-1 / 2} \pi \quad h \rightarrow m^{-1} \sqrt{m^{2}-\nabla^{2}} h
$$

leads to the desired helicity 1 canonical Lagrangian,

$$
L\left(T_{i}\right) \rightarrow \pi \dot{h}-\frac{1}{2}\left[\pi^{2}+h\left(m^{2}-\nabla^{2}\right) h\right] .
$$




\section{Acknowledgements}

This work was supported in part by Grants NSF PHY- 1266107 and DOE \# de-sc0011632. Collab-

oration with A. Waldron on [2] facilitated the present effort, as did composing help from J. Franklin and CPU help from G. Conrad.

\section{References}

[1] M Fierz, Helv Physica Acta 12, 3(1939).

[2] S.Deser and A.Waldron, Phys Lett B508, 347(2001).

[3] S.Deser, J. and S.Trubatch, Can J Phys 44, 1715(1966). 